(C2008 IEEE. Personal use of this material is permitted. However, permission to reprint/republish this material for advertising or promotional purposes or for creating new collective works for resale or redistribution to servers or lists, or to reuse any copyrighted component of this work in other works must be obtained from the IEEE 


\title{
Bragg Grating-Based Fiber-Optic Laser Probe for Temperature Sensing
}

\author{
Jharna Mandal, Suchandan Pal, Tong Sun, Kenneth T. V. Grattan, Andreas T. Augousti, and Scott A. Wade
}

\begin{abstract}
A novel Bragg grating-based fiber-optic laser probe for temperature sensing using erbium-doped fiber as the active gain medium is reported. The combination of a chirped grating and a normal grating was used to form the laser cavity to achieve temperature-tunable laser action over a wide measurement range. The laser probe used a metal sheath to enhance its mechanical strength and contain the normal grating at the sensing point. The temperature dependence of the wavelength of the laser probe gives a sensitivity of $12.01 \mathrm{pm} /{ }^{\circ} \mathrm{C}$ and a repeatability of $\pm 1.7^{\circ} \mathrm{C}$ from room temperature to $300{ }^{\circ} \mathrm{C}$.
\end{abstract}

Index Terms-Chirped grating, fiber Bragg gratings (FBGs), fiber-laser probe, temperature sensor.

\section{INTRODUCTION}

$\mathbf{F}$ IBER LASERS and amplifiers have been widely used in optical fiber communications systems for many years, but laser-based fiber-optic sensors are still relatively uncommon, in spite of the advantages of signal amplification in the optical, rather than in the electronic domain. Kim [1] has reviewed some of the fiber laser-based sensor devices produced over recent years, but as yet little work has been done on developing optical fiber laser-based systems for temperature sensing; for such measurements, some of the authors have used the fluorescence from rare-earth-doped fiber, especially using the thermal sensitivity of the decay time change [2]. Other relevant work has included a simple wavelength-matched Bragg grating laser cavity approach in rare-earth doped fiber to measure single-point strain and multipoint temperature, showing a linear temperature sensitivity of $0.011 \mathrm{~nm} /{ }^{\circ} \mathrm{C}$ [3]. Alavie et al. [4] have reported a multiplexed Bragg grating laser sensor system, measuring from $20{ }^{\circ} \mathrm{C}$ to $160{ }^{\circ} \mathrm{C}$, giving a sensitivity of $13.7 \mathrm{pm} /{ }^{\circ} \mathrm{C}$. For strain monitoring, recently, Kim et al. [5] have reported an intensitybased system for temperature-independent measurements using a single chirped grating.

The approach reported in this letter extends previous work to higher temperatures and uses the single-point probe approach, based on an infrared fiber laser, operating over a wide temperature range.

Manuscript received July 14, 2003; revised August 14, 2003. This work was supported by the Engineering and Physical Sciences Research Council of the U.K.

J. Mandal, S. Pal, T. Sun, and K. T. V. Grattan are with the School of Engineering and Mathematical Sciences, City University, London EC1V 0HB, U.K. (e-mail: k.t.v.grattan@city.ac.uk).

A. T. Augousti is with the School of Life Sciences, Kingston University, Kingston upon Thames, Surrey KT1 2EE, U.K.

S. A. Wade is with the Optical Technology Research Laboratory, Victoria University, Melbourne City MC, Victoria 8001, Australia.

Digital Object Identifier 10.1109/LPT.2003.820099

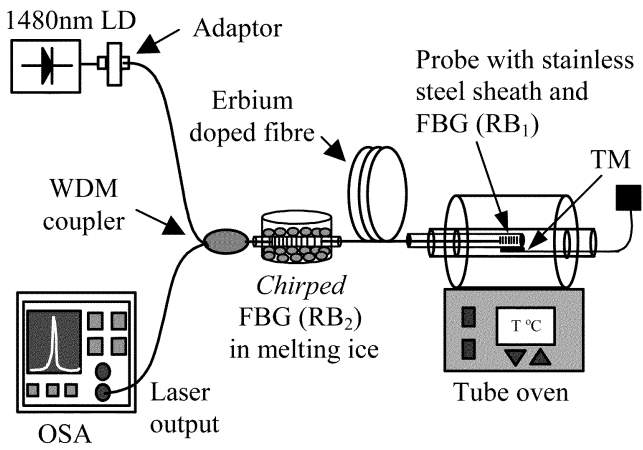

Fig. 1. Schematic diagram of an FBG-based laser probe sensor system. TM: thermocouple meter.

\section{EXPERIMENTAL ARRANGEMENT AND RESULTS}

The sensor system created is simple and robust (Fig. 1), using a commercial erbium-doped fiber of 350-400 ppm of erbium with a core diameter of $2.3 \mu \mathrm{m}$. This is fusion spliced to the fiber Bragg grating (FBG) reflectors (center wavelength of around $1535 \mathrm{~nm}$ ) to form the laser cavity and externally pumped by using a 1480-nm laser diode (LD) through a 1480/1550-nm wavelength-division-multiplexed coupler. The laser signal obtained is monitored using an optical spectrum analyzer (OSAHP86140A).

A schematic of the probe is shown in Fig. 1. It was constructed with the active part of the probe enrobed in a metal sheath for environmental protection. The laser probe consists of the normal grating $\left(\mathrm{RB}_{1}\right)$ which was placed in the center of the tube oven. The laser wavelength reflects its thermal response from the change in the center wavelength of the normal grating reflector $\mathrm{RB}_{1}$. This is due to the thermal expansion and the thermooptic coefficients of the fiber, which comprises the essential part of the temperature probe. A conventional laser system using two narrow-band Bragg reflectors could not be used, because of the change of the center wavelength of one of the FBGs in the probe. The use of a temperature-stabilized chirped Bragg grating as the second reflector solves the problem of creating an effective laser-based sensor-the potential wavelength range of the laser is greatly extended and with that the temperature measurement range of the device, from the $<200{ }^{\circ} \mathrm{C}$ maximum reported in previous work [3], [4]. In addition to creating a completely in-fiber based laser probe for temperature sensing, the second reflector was not a broad-band mirror [4], but a chirped grating, which makes the alignment of the laser configurations less sensitive to environmental effects-thus, simplifying the whole system.

The laser cavity length was optimized through several prior experiments and as a result fixed at $5 \mathrm{~m}$. This was a conve- 


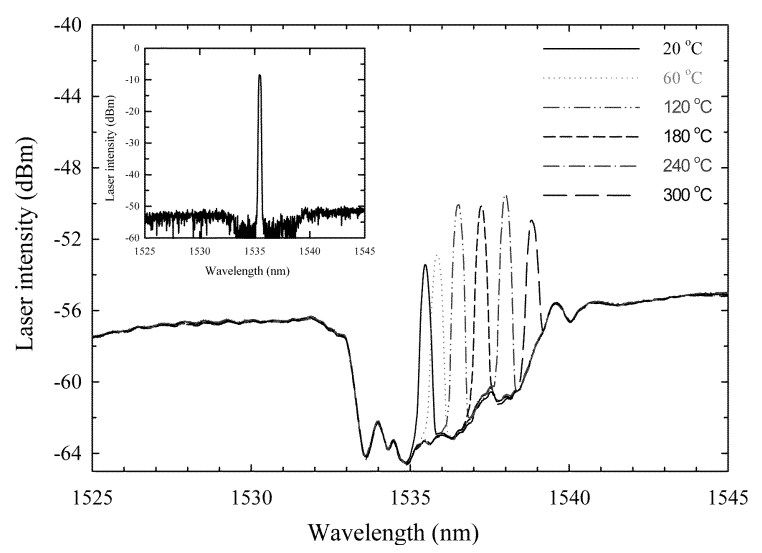

Fig. 2. Output spectrum of the fiber laser at various probe temperatures for a fixed pump power of $21 \mathrm{~mW}$. Inset shows the output spectrum at room temperature for an optimal pump power of $75 \mathrm{~mW}$.

nient length for a laser sensor probe - the sensitive grating in the probe assembly was, thus, some distance, the second reflector of the laser cavity.

Two different types of photosensitive fibers have been carefully selected in which to write the FBGs, using light from a $\mathrm{KrF}$ excimer laser (Braggstar-500, Tuilaser, AG) through appropriate phase masks, in order to optimize the range of the laser system. The first was Ge-doped fiber (from Nufern, Australia), into which was written the normal Bragg grating $\left(\mathrm{RB}_{1}\right)$, because of the long lifetime and high stability of gratings in this fiber at elevated temperatures [6]. Using the second B-Ge codoped fiber (from Fibercore Ltd., U.K.), the chirped Bragg reflector $\left(\mathrm{RB}_{2}\right)$ was written, because of its higher photosensitivity. The normal grating, $\left(\mathrm{RB}_{1}\right)$ had a reflectivity of more than $99 \%$ with a full-width at half-maximum (FWHM) bandwidth of $\sim 0.21 \mathrm{~nm}$ even after annealing at $320^{\circ} \mathrm{C}$ for $8 \mathrm{~h}$. To remove the unstable characteristics of the grating and also to achieve a stable and repeatable performance from the laser probe. Both the gratings were written with the choice of the Bragg wavelength ranges to lie within the fluorescence band of the erbium-doped fiber, to achieve temperature-tunable laser action, on which the sensor depends.

To evaluate the performance of the sensor, the chirped Bragg reflector $\left(\mathrm{RB}_{2}\right)$ was temperature stabilized (in a melting ice bath at $0{ }^{\circ} \mathrm{C}$ ) and the probe containing the normal Bragg reflector $\left(\mathrm{RB}_{1}\right)$ was placed in a thermostatically controlled tube oven (Carbolite-MTF12/38/400), with a K-type thermocouple in intimate contact for accurate calibration. The wavelength of the laser operation, the temperature-dependent measurand, was read out in this work using the OSA with a resolution bandwidth (RBW) of $0.2 \mathrm{~nm}$. The oven temperature was raised from room temperature to $300^{\circ} \mathrm{C}$, with an incremental step of $20^{\circ} \mathrm{C}$, and the sensor data were recorded at each temperature after stabilization. The spectrum of the fiber laser output was recorded as the temperature increased and the laser wavelength shifted to longer wavelengths, as is shown in Fig. 2. To show clearly that the laser wavelength does shift with temperatures over the broad-band range of the chirped grating, the pump power was fixed just above its threshold level. However, with a further increase of the pump power, the signal-to-noise ratio (SNR) of the system was easily improved (inset in Fig. 2), where the SNR was

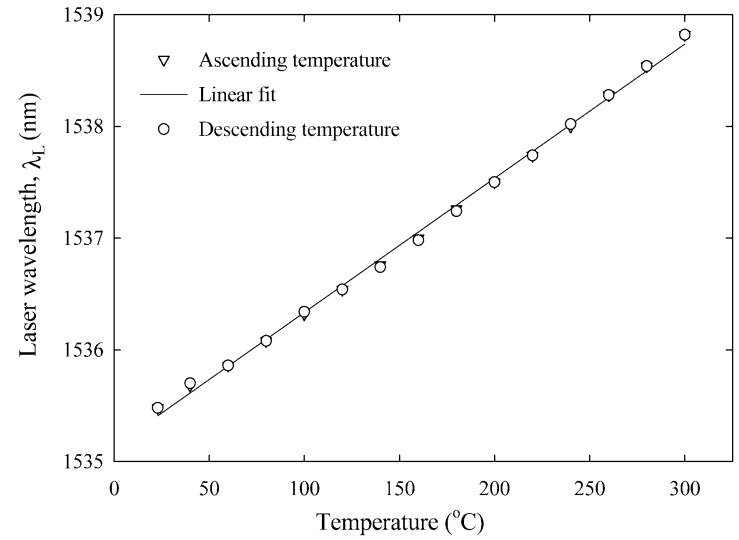

Fig. 3. Calibration of the sensor probe: The laser wavelength $\left(\lambda_{L}\right)$ versus temperature ( $T$ in degrees Celsius).

measured as approximately $47 \mathrm{~dB}$, higher than in some earlier laser work [7]. A typical probe calibration curve showing the laser wavelength $\left(\lambda_{L}\right)$ versus temperature ( $T$ in degrees Celsius) is shown in Fig. 3. The laser wavelength $\left(\lambda_{L}\right)$ with temperature can be determined through (1)

$$
\lambda_{L}=1535.13+0.01201 T
$$

where $T$ is the applied temperature to the normal grating $\left(\mathrm{RB}_{1}\right)$. The probe calibration was repeated several times with ascending and descending temperatures and the response was found to be highly repeatable within $\pm 0.02 \mathrm{~nm}$ from room temperature to $300{ }^{\circ} \mathrm{C}$, with almost no hysteresis being seen.

The linear response indicates that the sensitivity is essentially constant across the full measurement range from room temperature to $300{ }^{\circ} \mathrm{C}$, this being limited by the characteristics of the chirped grating $\mathrm{RB}_{2}$ (the FWHM bandwidth is $\sim 5.6 \mathrm{~nm}$ with a reflectivity of $75 \pm 5 \%$ ), which forms the second reflector of the laser cavity and also the choice of the Bragg wavelength of the normal grating $\mathrm{RB}_{1}$. From the characteristics of both the gratings and previous work on high-temperature grating fabrication [6], the upper limit of the measurement range of a system like this is expected to be no higher than $500{ }^{\circ} \mathrm{C}$, in order to allow a realistic lifetime for the probe before the grating "washes out." The repeatability of the measurement with the probe is $\pm 1.7^{\circ} \mathrm{C}$, considering the linear graph fitting calibration characteristic shown in Fig. 3 , with a and the sensitivity of $12.01 \mathrm{pm} /{ }^{\circ} \mathrm{C}$. This mainly arises from a combination of the maximum deviation of the sensor data at each temperature over the range from room temperature to $300{ }^{\circ} \mathrm{C}$ and the $\mathrm{RBW}$ of the OSA. The sensor could also be adapted for measurements below room temperature using a slightly different wavelength range of the probe grating $\left(\mathrm{RB}_{1}\right)$ with appropriate material substrates [8], e.g., employing Teflon, as the bare silica fiber has a negative thermal expansion coefficient below $\sim 150 \mathrm{~K}$ [9]. Further detailed studies on the laser-based cryogenic measurements are interesting and will be the subject of future work.

The sensor system has also been tested by reversing the measurement point in the laser cavity-the chirped grating was placed in the tube oven and the normal grating was kept at the ambient temperature. Under such circumstances, the laser wavelength should not change with temperature as it would be 


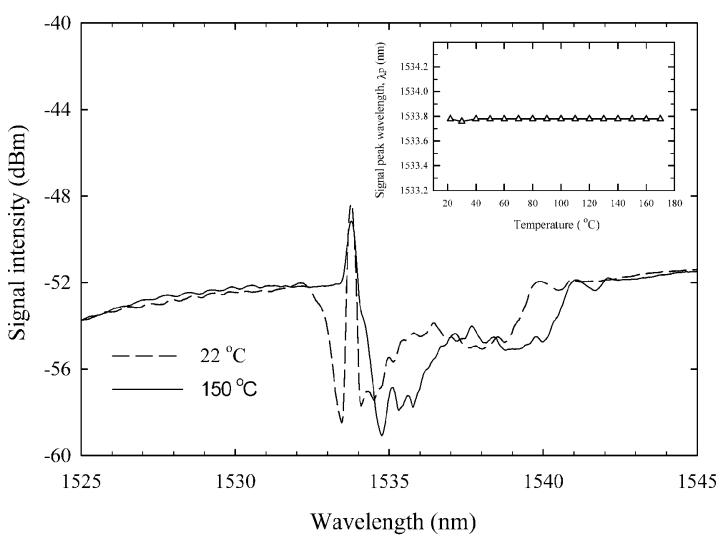

Fig. 4. Response of the system where the reverse measurement configuration is used and the chirped grating is placed in the calibration oven. Inset shows the response of the signal peak wavelength $\left(\lambda_{p}\right)$ to the temperature change.

determined by the normal grating held at a fixed temperature. The experimental results for the chirped grating, over the range of oven temperatures used, are shown in Fig. 4. The spectra recorded reveal that the laser wavelength is unchanged but the spectrum changes slightly. The effect is seen more fully from the inset in Fig. 4, where the expected flat line confirms that the laser wavelength is determined only by the wavelength of the normal grating irrespective of the temperature variation of the chirped grating, owing to its much narrower FWHM bandwidth $\sim 0.17 \mathrm{~nm}$ (with reflectivity of $97 \%$ after annealing at $515^{\circ} \mathrm{C}$ ) compared with that of the chirped grating bandwidth $\sim 5.6 \mathrm{~nm}$. This test result confirms that the configuration in Fig. 1 operates as a true laser sensor where the laser wavelength is effective as the temperature-dependent measurand (Fig. 3). The reverse measurement point approach is not suitable for sensing applications as the laser signal peak wavelength does not vary (inset in Fig. 4) and, thus, the temperature data cannot be obtained from the laser wavelength.

\section{CONCLUSION}

A simple laser-based FBG temperature sensor probe, exploiting the characteristics of FBGs in specially chosen fibers used for the laser cavity, both in terms of the wavelength of op- eration and the long lifetime at elevated temperatures, has been reported. The reverse measurement configuration of the laser cavity was used to show that when the normal grating $\left(\mathrm{RB}_{1}\right)$ was allowed to respond to different temperatures, the device does work as a laser-based sensor. The laser-based sensor system offers a better SNR than the fluorescence-based temperature sensors previously reported by us [2], is convenient, stable, and highly repeatable, and also relatively inexpensive to fabricate. This work has demonstrated a new approach to the use of high temperature FBG-based laser probes for high-temperature wide range sensing the applications. Work is continuing to evaluate the use of other fibers with other dopants, and to enlarge the measurement range as well to extend the concept to a multiplexed sensing scheme, and thus, the range of uses, e.g., fire alarm systems, furnace monitoring, and microwave sensing.

\section{REFERENCES}

[1] B. Y. Kim, "Fiber lasers in optical sensors," in Optical Fiber Sensor Technology, 1st ed, K. T. V. Grattan and B. T. Meggitt, Eds, London, U.K.: Chapman \& Hall, 1998, vol. 2, pp. 99-115.

[2] Z. Y. Zhang, K. T. V. Grattan, A. W. Palmer, B. T. Meggitt, and T. Sun, "Characterization of erbium-doped intrinsic optical fiber sensor probes at high temperatures," Rev. Sci. Instrum., vol. 69, no. 8, pp. 2924-2929, 1998 .

[3] G. A. Ball, W. W. Morey, and P. K. Cheo, "Single- and multipoint fiberlaser sensors," IEEE Photon. Technol. Lett., vol. 5, pp. 267-270, Feb. 1993.

[4] A. T. Alavie, S. E. Karr, A. Othonos, and R. M. Measures, "A multiplexed Bragg grating fiber laser sensor system," IEEE Photon. Technol. Lett., vol. 5, pp. 1112-1114, Sept. 1993.

[5] S. Kim, J. Kwon, S. Kim, and B. Lee, "Temperature-independent strain sensor using a chirped grating partially embedded in a glass tube," IEEE Photon. Technol. Lett., vol. 12, pp. 678-680, June 2000.

[6] S. Pal, J. Mandal, T. Sun, K. T. V. Grattan, M. Fokine, F. Carlsson, P. Y. Fonjallaz, S. A. Wade, and S. F. Collins, "Characteristics of potential fibre Bragg grating sensor-based devices at elevated temperatures," Meas. Sci. Technol., vol. 14, pp. 1131-1136, June 2003.

[7] A. D. Kersey and W. W. Morey, "Multiplexed Bragg grating fibre-laser strain-sensor system with mode-locked interrogation," Electron. Lett., vol. 29, no. 1, pp. 112-114, 1993.

[8] T. Mizunami, H. Tatehata, and H. Kawashima, "High-sensitivity cryogenic fibre-Bragg-grating temperature sensors using teflon substrates," Meas. Sci. Technol., vol. 12, pp. 914-917, 2001.

[9] S. Gupta, T. Mizunami, T. Yamao, and T. Shimomura, "Fiber Bragg grating cryogenic temperature sensors," Appl. Opt., vol. 35, no. 25, pp. 5202-5205, 1996. 